\title{
Valoración del diseño estratégico de una compañía hotelera a través de indicadores cualitativos y cuantitativos
}

\author{
Dinaidys Gómez-Selemeneva \\ digomsel@upo.es \\ Universidad Pablo de Olavide \\ Idalia Romero Lamorú \\ idalia@fec.uh.cu \\ Universidad de La Habana \\ María Solís Corvo \\ maria@fec.uh.cu \\ Universidad de La Habana
}

\section{Resumen}

El proceso de diseño estratégico y la posterior evaluación de resultados así como su retroalimentación y control constituyen momentos claves en la gestión de una organización. Esta es por tanto una temática de gran relevancia y actualidad en todas las publicaciones de esta área temática, en especial los trabajos empíricos que aporten información tanto cuantitativa como cualitativa del desempeño de empresas de sectores dinámicos como es el caso del turismo. Es por ello que el presente artículo centra su atención en la valoración de la efectividad de las diferentes estrategias: genéricas, de crecimiento y de diversificación de una compañía hotelera de prestigio en al área del Caribe a través de los indicadores de desempeño financiero y otros cualitativos. Los resultados que se presentan corresponden al $100 \%$ de la población objeto de estudio, todo lo cual posibilitó la información necesaria para detectar las principales desviaciones respecto al planteamiento estratégico, así como las posibles alternativas de mejora del rendimiento y la competitividad futura de las diferentes instalaciones hoteleras. Con lo que se aporta una investigación empírica de un destino turístico en crecimiento y consolidación, así como una también una metodología generalizable para el diagnóstico y diseño estratégico de las organizaciones

Palabras clave: Planificación estratégica, destino turístico, ocupación hotelera, capacidades dinámicas, desempeño financiero. 


\section{Introducción}

Es reconocido por los referentes en la temática de estrategia la importancia de la evaluación del desempeño estratégico de las organizaciones es un elemento clave para el sostenimiento de la posición que alcanzan en el mercado y para la rentabilización del negocio en el tiempo (Mintzberg \& Quinn, 1997; Mintzberg \& Lampel 1999; Porter, 1991). Además, en la literatura se percibe el peso que ha ido ganando, cada vez más en el análisis y debate académico, el tema relacionado con la implantación y control de las estrategias respecto a la fase de planificación lo cual era el centro de atención en los primeros momentos (Powell, 1992, Siu-Loon, 2007). Es por ello que cada vez de manera más creciente los estudios de esta disciplina buscan profundizar en aquellos aspectos del Diseño, la Formulación y la Retroalimentación de las alternativas estratégicas seguidas por una organización (Allen \& Helms, 2006; Grant, 2004).

Puesto que cada sector de actividad tiene sus particularidades la concepción de la estrategia a asumir y su planificación y control, existen un gran número de trabajos empíricos. En el caso de la actividad hotelera diversos autores han encausado la investigación hacia variables de resultado estratégico tales como el Performance (Garrigo's \& Palacios, 2005; Pappas et al., 2007; Tavitiyaman et al., 2012; Reichel \& Haber, 2005) la satisfacción de los clientes ( Ekinci, 2004; Kim et al, 2012) o el impacto en la economía local (Claver-Cortés et al, 2009; Romero, 2000). En todos estos trabajos se han realizado importantes aportaciones quedando, sin embargo importantes Gaps, susceptibles de ser analizados como por ejemplo las variables para la medición de la efectividad de las estrategias implantadas (Allen \& Helms, 2006; Sainaghir, 2010) o el énfasis del tratamiento teórico de la implantación ha estado más dirigido al esclarecimiento del soporte a la estrategia y a la explicación de los problemas del fracaso de la implantación de la estrategia (Akan; 2006; Chandler, 2003).

En general, aunque abundan los trabajos empíricos que abordan el caso las estrategias Genéricas seguidas por Cadenas Hoteleras, sí que se aprecia una necesidad de hacer extensivos estos estudios a destinos turísticos emergentes donde esta actividad tenga un especial dinamismo en los últimos 10 años como es el caso del sector en el la región del Caribe y en especial en la Habana, Cuba (Bailey, 2008; Jonsson, \& Devonish, 2009), con lo cual la investigación que se presenta hará una necesaria y útil contribución a través de la metodología propuesta basada en indicadores cualitativos y cuantitativos que contribuyan a la evaluación y control de las estrategias desplegadas por la Compañía Habaguanex S.A.

\section{Fundamentación teórica}

"La Dirección Estratégica puede concebirse como una estructura teórica para la reflexión acerca de las grandes opciones de la empresa, reflexión que se fundamenta en una nueva cultura organizacional y una nueva actitud de la dirección, donde hay que ir al encuentro del entorno. Es también un intento de mejorar la dirección y la gestión de una organización, utilizando las estrategias para guiar sus acciones, pero 
integrando las nociones de preparación de la puesta en marcha y la asignación de recursos" (Menguzato y Renau, 1991). En cambio la Planificación estratégica constituye un importante paso de avance en cuanto incluye en su análisis al entorno y propone alternativas estratégicas además de que incorpora a los directivos en la elaboración de las mismas.

El éxito del comportamiento estratégico de una cadena hotelera depende de dos cuestiones importantes, por un lado, el atractivo del sector en el que actúa y por otro los factores determinantes de una posición competitiva relativa dentro del mismo. Además de las características estructurales principales del negocio hotelero es importante adicionar otras que complementan el análisis del posicionamiento en la formulación de estrategias. Estas son: el carácter cíclico y estacional con alta dependencia del ciclo de la economía, alto nivel de atractivo para la inversión y financiación externa, dependencia de un soporte físico o planta hotelera, explotación y resultados en el largo plazo y altos volumen de costos indirectos (Romero, 2000). Estas variables influyen el proceso de implementación y los resultados que se obtienen, en el proceso de diagnóstico y control para este sector de actividad.

Numerosos trabajos atribuyen una mayor relevancia al control estratégico como parte del proceso de dirección estratégica de la empresa (Grant, 2004; Hunt et al, 2009; Julian \& Scifres, 2002; Menguzzato y Renau, 1991; Powell, 1992; Rumel, 1994). Específicamente el trabajo de Grant, esclarece el concepto de control estratégico y la diferenciación entre planificación y control. El control estratégico abarca tanto el control de la implantación como el control de la estrategia. Según sus puntos de vista la planificación fija los objetivos y planes concretos para una actividad.

El control observa los resultados, los compara con los objetivos y toma las medidas oportunas para corregir las posibles desviaciones, está encaminado a la revisión y seguimiento del propio proceso de implantación o puesta en marcha (Navas y Guerra, 2002).Es común el papel que le otorgan los autores a la interacción entre las fases de formulación e implantación, sin embargo no abundan los trabajos dirigidos a la medición de la efectividad de las estrategias implantadas como vía de retroalimentación y corrección del diseño estratégico. El énfasis del tratamiento teórico de la implantación ha estado más dirigido al esclarecimiento del soporte a la estrategia y a la explicación de los problemas del fracaso de la implantación de la estrategia (Navas y Guerra, 2002).

En la actividad hotelera esta dimensión de análisis ha sido abordada en trabajos empíricos los cuales aportan variables de gestión claves. En la tabla 1 se ha realizado un análisis de la bibliografía, el cual muestra la creciente importancia que se le ha atribuido al conocimiento de aquellos factores que determinan la preferencia, atractivo o competitividad en la gestión de la oferta turística (Ortigueira-Sánchez y GómezSelemeneva, 2012). Con lo cual el proceso de Diseño, implementación y control estratégico estará en la valoración del papel que tiene cada uno de estos elementos en la concepción de la estrategia de la organización. 
Tabla 1. Variables Claves en la Planificación y Diseño de las estrategias Turísticas.

\begin{tabular}{|c|c|c|c|c|c|c|c|c|c|}
\hline \multirow[t]{2}{*}{ AUTOR } & \multicolumn{9}{|c|}{ VARIABLES DE INVESTIGACIÓN } \\
\hline & Alojamiento & Accesibilidad & $\begin{array}{l}\text { Calidad } \\
\text { / precio }\end{array}$ & Seguridad & $\begin{array}{l}\text { Interacción } \\
\text { social }\end{array}$ & Autenticidad & Cultura & Gastronomía & $\begin{array}{l}\text { Actividad } \\
\text { nocturna }\end{array}$ \\
\hline $\begin{array}{l}\text { Baloglu } \\
\text { (1997) }\end{array}$ & $x$ & $x$ & $x$ & $x$ & $x$ & $x$ & $x$ & $x$ & $x$ \\
\hline $\begin{array}{l}\text { Baloglu \& } \\
\text { McCleary } \\
\text { (1999) }\end{array}$ & $x$ & $x$ & $x$ & $x$ & & & $x$ & $x$ & $\mathbf{x}$ \\
\hline $\begin{array}{l}\text { Crompton \& } \\
\text { Fakeye } \\
(2006)\end{array}$ & $x$ & & $x$ & & & & $x$ & $\mathbf{x}$ & $x$ \\
\hline $\begin{array}{l}\text { Crompton } \\
\text { 2011) }\end{array}$ & $\mathrm{x}$ & & $\mathrm{x}$ & $x$ & & $x$ & & $\mathbf{x}$ & $x$ \\
\hline $\begin{array}{l}\text { Echtner \& } \\
\text { Ritchie } \\
\text { (1993) }\end{array}$ & $x$ & $x$ & $\mathbf{x}$ & $x$ & $\mathbf{x}$ & & $x$ & $\mathbf{x}$ & $x$ \\
\hline $\begin{array}{l}\text { Kim,et a.I } \\
\text { (2012) }\end{array}$ & $x$ & $x$ & $x$ & $x$ & $x$ & $x$ & $x$ & & \\
\hline $\begin{array}{l}\text { McLennan } \\
\text { et al.( } 2010)\end{array}$ & $x$ & $x$ & & $x$ & $x$ & $x$ & $x$ & $x$ & $\mathbf{x}$ \\
\hline $\begin{array}{l}\text { Opperman } \\
\text { (1996) }\end{array}$ & $x$ & & $x$ & $x$ & & & $x$ & $\mathbf{x}$ & $x$ \\
\hline $\begin{array}{l}\text { Amstrong } \\
\text { (2007) }\end{array}$ & $\mathrm{x}$ & $\mathrm{x}$ & $\mathrm{x}$ & $x$ & $\mathrm{x}$ & & $\mathbf{x}$ & $\mathrm{x}$ & \\
\hline $\begin{array}{l}\text { Schroeder } \\
\text { (1996) }\end{array}$ & $x$ & & $\mathbf{x}$ & & & $x$ & & $x$ & $x$ \\
\hline
\end{tabular}

Fuente: Elaboración propia.

\section{Metodología}

El propósito de la presente investigación es abordar la formulación y control de las estrategias para el caso de una compañía turística cubana denominada Habaguanex S.A. Aportándose un análisis empírico en el que se vincula la planificación con la retroalimentación del proceso enfocado a la efectiva gestión de las variables claves mencionadas en el epígrafe anterior.

A todo lo cual se añadirá la evaluación de los principales indicadores de desempeño: Ocupación y Rendimiento, de la actividad económica que se desarrolla en instalaciones de esta cadena durante los últimos 5 años. El proceso de investigación por el que se transitó se presenta en la figura 1.

Como parte de la investigación empírica se aplicaron entrevistas semiestructuradas al $100 \%$ de la población objeto de estudio correspondiente a puestos de dirección de la compañía y a los directores de las instalaciones hoteleras. En tanto la información financiera y de desempeño fue la aportada por la Compañía y por el Ministerio de Turismo de Cuba. Por último el análisis estratégico, y las propuestas de reconfiguración de las estrategias futuras (su adecuación, adaptabilidad y factibilidad) fueron elaboradas por un grupo de expertos integrados por académicos, directivos de la compañía y staff de apoyo así como directores de calidad de las instalaciones Hoteleras.

Posteriormente a la entrevista preliminar y desestructurada, se aplicó un cuestionario durante los meses de diciembre de 2011 a marzo de 2012, el cual tenía como finalidad 
evaluar la relación entre el camino estratégico seguido y los resultados finales obtenidos En el mismo además se analiza el peso otorgado a las variables claves en este diseño estratégico. Así como se plantean interrogantes sobre las alternativas estratégicas.

Figura 1. Propuesta metodológica para el estudio empírico desarrollado

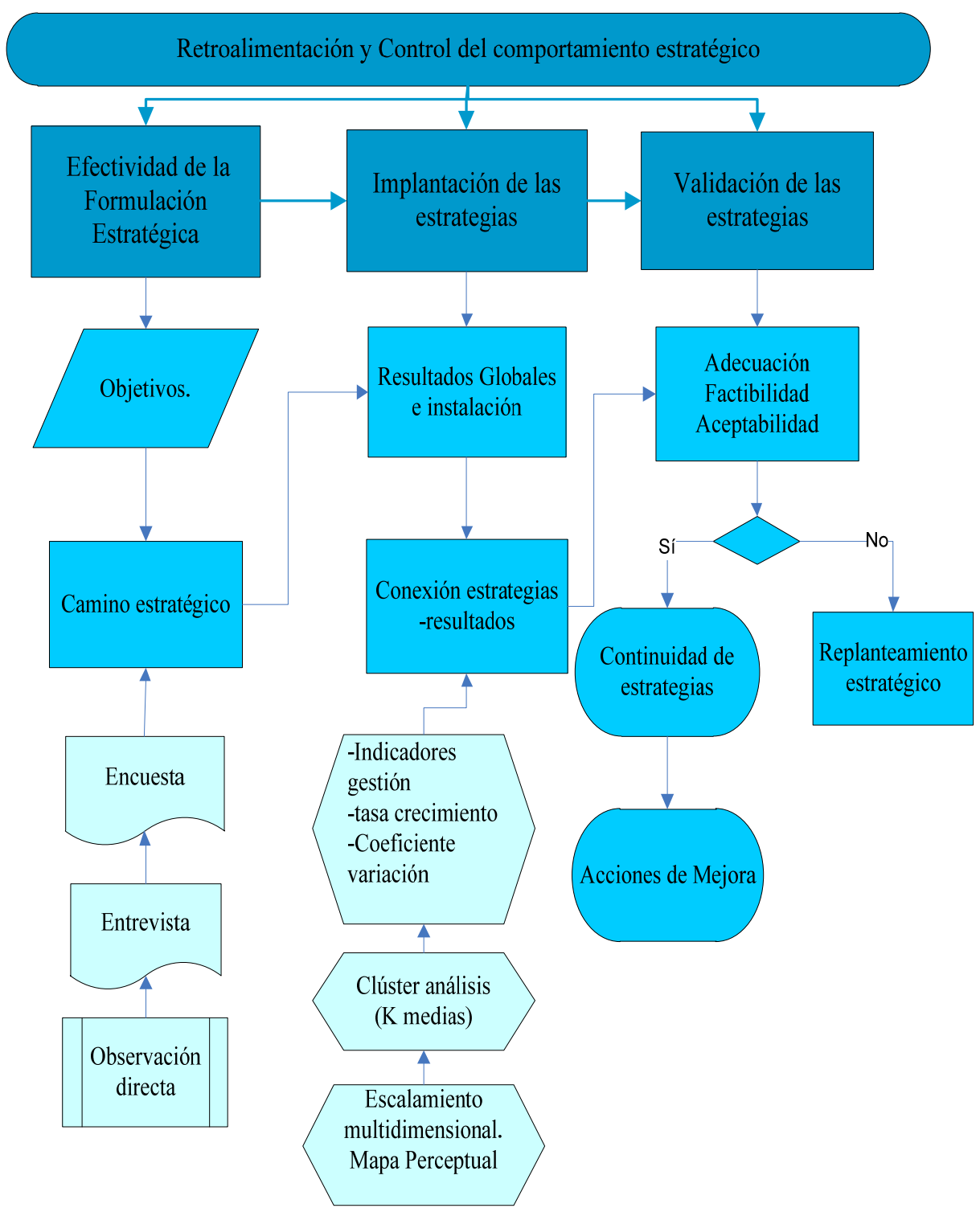

Fuente: Elaboración propia

Las tablas 2 y 3 corresponden a las respuesta de los encuestados en lo en cuanto a la importancia concedida a cada uno de los factores claves y a las alternativas estratégicas. 
Tabla 2. Significación de la escala.

\begin{tabular}{lcc}
\hline \multicolumn{1}{c}{ Escalas } & W de Kendall & Sig. asintót. \\
\hline Objetivos y Resultados & 0,341 & 0,00 \\
Factores de éxito & 0,538 & 0,00 \\
Estrategias & 0,618 & 0,00 \\
\hline
\end{tabular}

Alfa de Cronbach de 0,859 (N.18)

Tabla 3: Matriz de Correlaciones factores claves de éxito estratégico

\begin{tabular}{llccccccccc}
\hline & VARIABLE & Mean & Min & Max & $\mathbf{1}$ & $\mathbf{2}$ & $\mathbf{3}$ & $\mathbf{4}$ & $\mathbf{5}$ & $\mathbf{6}$ \\
\hline $\mathbf{1}$ & Identidad y personalidad & 4.611 & 4 & 5 & 1.000 & & & & & \\
$\mathbf{2}$ & Atención personalizada & 3.722 & 1 & 5 & 0.6269 & 1.000 & & & & \\
$\mathbf{3}$ & Seguridad & 4.055 & 2 & 5 & 0.4238 & 0.6787 & 1.000 & & & \\
$\mathbf{4}$ & Oferta gastronómica & 2.666 & 1 & 5 & -0.1374 & 0.0522 & 0.0735 & 1.000 & & \\
$\mathbf{5}$ & Atractivos nocturnos & 1.5 & 1 & 5 & 0.0562 & 0.1923 & 0.1504 & 0.5948 & 1.000 & \\
$\mathbf{6}$ & Relación calidad-precio & 2.777 & 1 & 4 & -0.3412 & -0.0056 & 0.0873 & 0.5103 & 0.4495 & 1.000 \\
\hline Alfa de Cronbach .64 (N.18) & & & & & & & & &
\end{tabular}

Es importante destacar que en el presente artículo es solo un aparte del desarrollo del Modelo propuesto pata la implementación de la metodología por lo que no aparecerán algunas de sus partes, ni de los análisis desarrollados, aunque todos ellos contribuyen a las propuestas y conclusiones a las que se arriban.

\section{Resultados}

Hoteles Habaguanex es una de las líneas de negocio de la Compañía Turística Habaguanex S.A, creada en el año 1994; como parte del empeño de la Oficina del Historiador de la Ciudad de lograr una explotación turística planificada y sustentable del Centro Histórico de La Habana e integrar esfuerzos de restauración y rehabilitación bajo un enfoque de vialidad económica dentro de la cual la actividad turística desempeña un papel dinamizador y generador de recursos (Leal, 2004). Es por todo esto que surge el negocio hotelero constituyendo en el año 2011 el $7 \%$ de los ingresos (gráfico 1). 
Gráfico 1. Tasa de crecimiento anual de Hoteles Habaguanex.

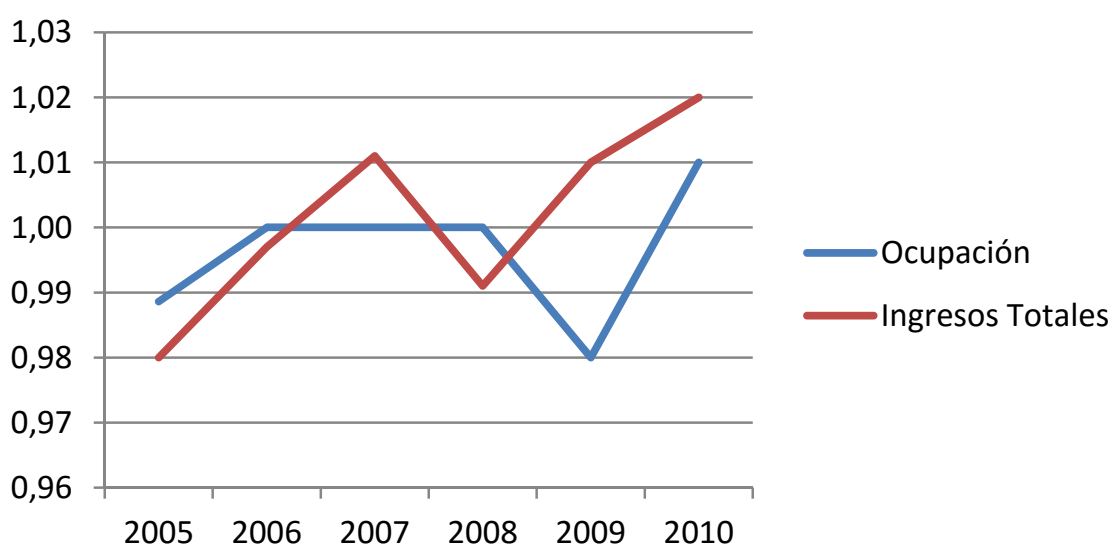

Fuente: Elaboración propia a partir de datos ofrecidos por la cadena

El negocio hotelero desde sus inicios concibe una estrategia de crecimiento por vía de especialización en el alojamiento, básicamente en alojamiento, sin embargo ya en los tres primeros años de explotación crea instalaciones nuevas que desde su apertura diversifican el alojamiento hacia el comercio y la restauración. La estrategia de diversificación relacionada tiene su auge en el año 2008 , en el que el $87 \%$ de las instalaciones asumen dicho comportamiento.

Desde el punto de vista competitivo es claro el enfoque general del grupo hacia la diferenciación, mediante la potenciación de características peculiares del diseño, confort y servicio, lo que define su estrategia como alta Segmentación, ofreciendo un producto que aporta alto valor añadido a los clientes a precios altos.

La implantación de la misma contiene importantes atributos de gran atractivo como destino: un producto turístico en un entorno único que revela alto valor arquitectónico, cultural e histórico secular y la seguridad. La Filosofía se basa en brindar una oferta exclusiva, orientada fundamentalmente a satisfacer las necesidades de un segmento de mercado internacional interesado en la historia y tradiciones cubanas. Su diseño se perfila mediante hoteles pequeños, íntimos, decoración con temáticas distintivas, entre las que se encuentran orígenes, tradición tabacalera, arte marítimo y de la herencia cultural española, norteamericana y caribeña.

Para el cumplimiento de los propósitos de la investigación se utilizan los indicadores cuantitativos: ingreso total, ingreso por habitación existente, utilidades y ocupación, por instalaciones hoteleras, que abarcan el periodo 2005-2010 tanto a nivel corporativo como de las instalaciones. Todo lo cual posibilita determinar el posicionamiento que alcanza cada uno de los hoteles en el período 2005-2010 (Gráfico 2). La distribución espacial en el posicionamiento de las instalaciones muestra distancias de forma triangular. 
Gráfico 2. Mapa de Ubicación de las instalaciones por conglomerado

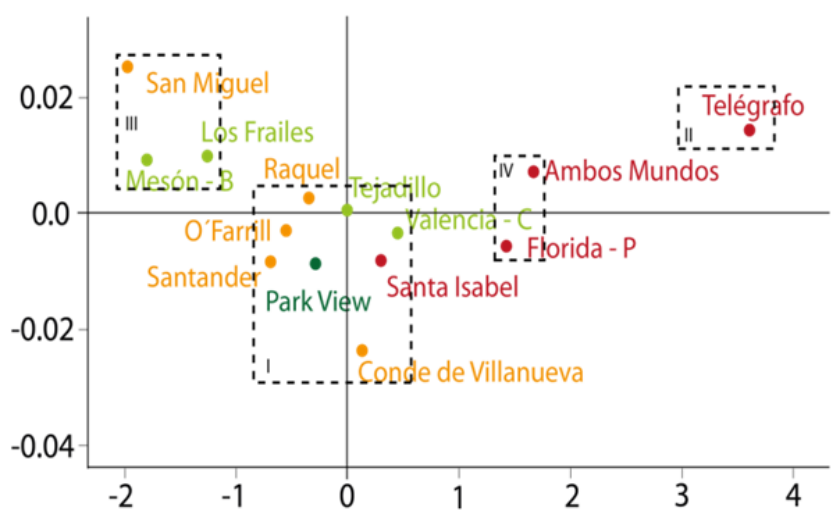

Fuente: Indicadores cuantitativos de gestión Adaptado salida SPSS

Con el objetivo de profundizar en las causas que originan las brechas existentes en dicho posicionamiento, se determina el coeficiente de variación (Cv) de los resultados de los indicadores: ingresos, ocupación y utilidades, para cada una de las instalaciones en el período que se analiza, mediante la fórmula: $\mathrm{Cv}=\mathrm{S} / \mathrm{Xmedia}$.

A través del coeficiente de variación, se refleja la heterogeneidad en su comportamiento; oponiéndose la mayor estabilidad de los indicadores de ocupación, es decir alojamiento, a la mayor inestabilidad de las utilidades, estos resultados podrían tener como principal causante el hecho de una aplicación de estrategias diferentes por instalaciones hoteleras (Gráfico 3). Una primera iteración podría sugerir la influencia del tamaño de las instalaciones en las distancias como factor explicativo entre los más alejados, sin embargo, no podemos afirmar que el tamaño es suficiente para explicar los resultados.

Gráfico 3. Coeficiente de Variación de ingresos, utilidades y ocupación en el período

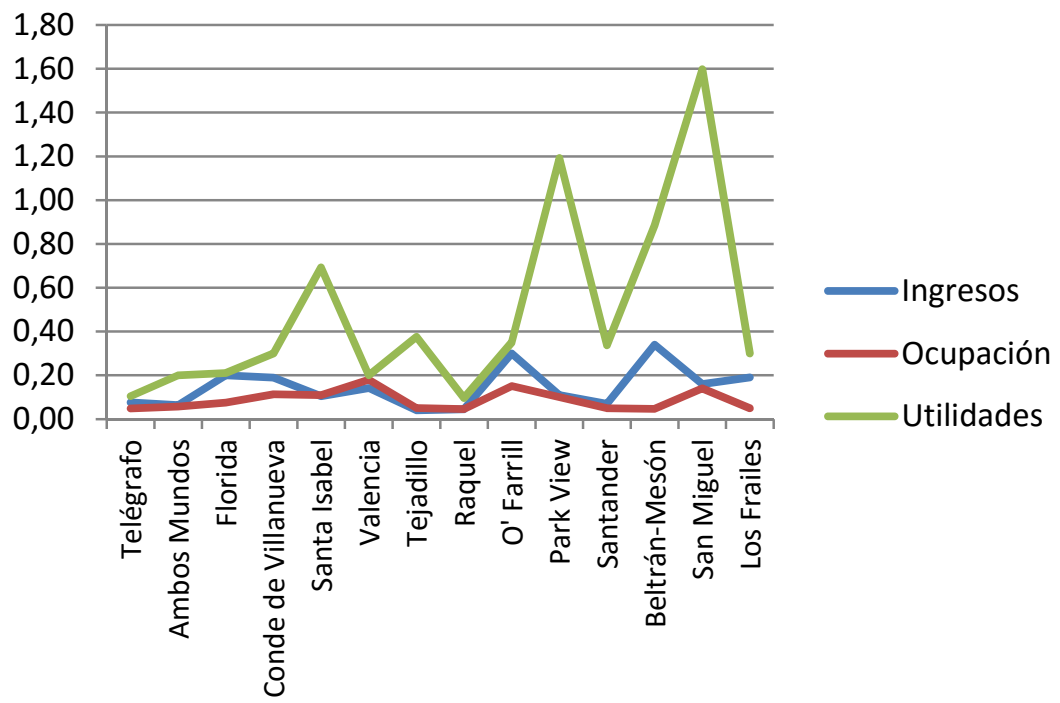

Fuente: Elaboración propia a partir de datos ofrecidos por Habaguanex

El análisis por instalaciones, refleja mayores brechas en el comportamiento de la variación en los conglomerados 1 y 3 . Los picos que se observan muestran la inestabilidad en los resultados, que se hace más crítica en los hoteles Park View y San 
Miguel, los cuales sólo llevan a cabo la estrategia de especialización mediante el alojamiento.

De igual forma, los hoteles que sólo combinan el alojamiento con una de las ramas de la diversificación presentan resultados inestables, lo que en menor medida; tal es el caso del Santa Isabel, O'Farril, Tejadillo y Santander; a diferencia de los hoteles que combinan dos o más estrategias, como el Florida, Valencia, Conde de Villanueva, Ambos Mundos, Los Frailes y Telégrafo, cuyos indicadores describen estabilidad.

Para la valoración de alternativas estratégicas futuras, los indicadores de significación de escalas se muestran a continuación en la tabla 4.

Tabla 4. Estadísticos descriptivos variables estratégicas

\begin{tabular}{llccccc}
\hline & VARIABLE & N & Mean & Min & Max \\
\hline 1 & Incentivar turismo histórico cultural & 18 & 4.611 & 4.611 & 3 & 5 \\
2 & Mayores ingresos para la compañía & 18 & 4.277 & 4.277 & 2 & 5 \\
3 & Desarrollo turismo histórico-cultural & 18 & 4.611 & 4.611 & 3 & 5 \\
4 & Desarrollo del entorno urbano & 18 & 4.5 & 4.5 & 3 & 5 \\
5 & Segmentación temática & 18 & 4 & 4 & 2 & 5 \\
6 & Marca propia & 18 & 3.833 & 3.833 & 1 & 5 \\
\hline Alfa de Cronbach .758 & & & & &
\end{tabular}

Los hoteles del grupo manifiestan un comportamiento heterogéneo debido a que todos los hoteles no asumieron por igual las estrategias globales de Hoteles Habaguanex. Podemos afirmar que en la actualidad existen particularidades que tipifican las actuaciones estratégicas de las instalaciones y son las que conducen a los resultados alcanzados. El "Reloj Estratégico"(figura 2), refleja el grado de concreción de la estrategia diseñada por la compañía, la valoración de los resultados y efectividad de la misma, lo cual era un objetivo importante de la investigación. Además se establecen alternativas estratégicas futuras que tengan en cuenta tanto los factores claves estudiados como el camino a seguir para su consecución.

La estrategia competitiva a nivel de grupo hotelero comprendida de manera general como diferenciación segmentada, presenta especificidades. Si se observa la figura 2 , los hoteles Santa Isabel, Telégrafo, Ambos Mundos y Florida Prado Ameno, se colocan en la ruta 5 , siguiendo una estrategia de alta diferenciación segmentada, ya que son hoteles de altos precios y un alto valor añadido. En tanto en la ruta 4 se ubican los hoteles Conde de Villanueva, Raquel, San Miguel, Santander y O'Farril, con precios moderados y un producto diferenciado. En la ruta 3 se encuentran los hoteles Mesón-Beltrán, Valencia- Comendador, Los Frailes y Tejadillo; hoteles de un precio bajo, pero de un valor añadido alto. Por último, en la ruta 2, se halla el hotel Park View, que es el hotel de precios más bajos del grupo y un valor añadido percibido medio. 
Figura 2.Reloj Estratégico.

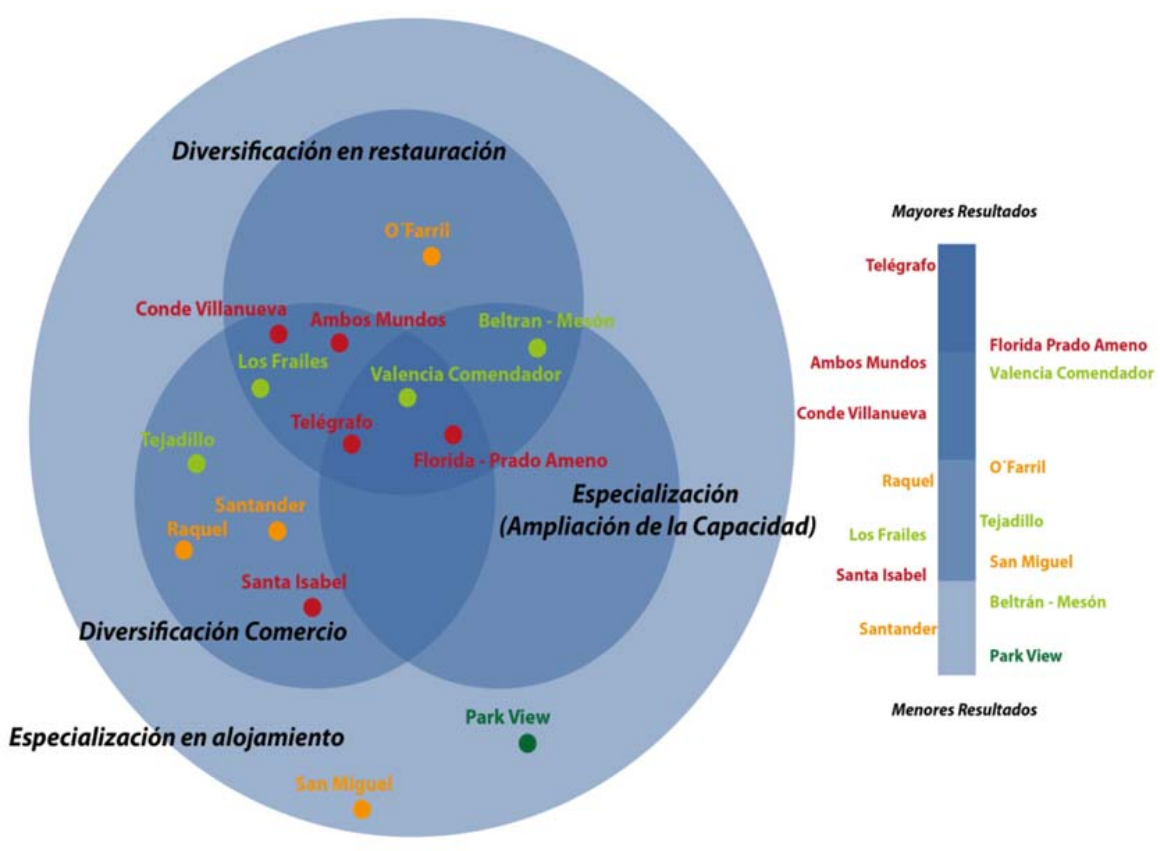

Fuente: Elaboración Propia

Los resultados no se aprecian correlacionados con las estrategias competitivas genéricas que guardan relación con la escala de colores del Reloj Estratégico. También se confirma que la correlación favorable entre los hoteles que alcanzan los mejores resultados se concentran en aquellos hoteles que con independencia de la estrategia genérica combinan la estrategia de diversificación no siendo así para las estrategias de especialización.

\section{Conclusiones}

El posicionamiento estratégico que se revela, mediante las técnicas utilizadas (análisis de Clúster de $\mathrm{K}$ medias, el Escalamiento Multidimensional y Coeficiente de variación por instalaciones), refleja la conexión de los resultados con las estrategias asumidas demostrándose las bondades que en dicho resultados tiene el despliegue de la diversificación frente a la especialización del alojamiento.

La efectividad en el diseño de las estrategias se constata mediante el análisis de los indicadores de la gestión. Además, la estabilidad del desempeño muestra vínculo causal con las estrategias asumidas lo cual era un objetivo de la investigación. Sin embargo como resultado del estudio realizado, el análisis e interpretación de los datos cuantitativos surgen cuestiones que suscitan su discusión y debate por parte de los investigadores.

La investigación devela el camino estratégico de Hoteles Habaguanex para su inserción en el mercado turístico internacional mediante la alta diferenciación segmentada en la explotación del turismo histórico cultural, por lo que se deberá ppromover mayores actividades gastronómicas, culturales y espectáculos que integren al visitante en el maravillo entorno que rodea la Habana Vieja. 
Como continuidad de esta investigación se propone el seguimiento al rediseño estratégico de la compañía en la que se potencien los factores claves enunciados en la literatura y corroborados por los expertos como es el caso de la Identidad y personalidad cosa que ha estado implícita en la concepción de un producto diferenciado que potencie los valores, costumbres e historia de la Isla de Cuba, así como el tema de la Seguridad. Además se deberán establecer criterios de control especialmente para los relacionados con la relación calidad-precio.

Como importante aportación estimamos que la Metodología desarrollada amplía el horizonte de los mecanismos a utilizar en la fase de implantación, retroalimentación y control estratégico. Este estudio, además, abre nuevas líneas de investigación para el diagnóstico estratégico de compañías en destinos semejantes que busquen ampliar la imagen proyectada de Destinos de Sol y Playa a otros atractivos patrimoniales, históricos y culturales, como es el caso de los llamados "Hoteles Boutique", el norte de Europa, los "Hoteles con Encanto" en Latinoamérica y los "Paradores" en España.

\section{Referencias bibliográficas}

Akan, O., Allen, R. S., Helms, M. M., \& Spralls III, S. A. (2006). Critical tactics for implementing Porter's generic strategies. Journal of Business Strategy, 27(1), 43-53.

Allen, R. S., \& Helms, M. M. (2006). Linking strategic practices and organizational performance to Porter's generic strategies. Business Process Management Journal, 12(4), 433-454.

Bailey, N. (2008). The challenge and response to global tourism in the post-modern era: the commodification, reconfiguration and mutual transformation of Habana Vieja, Cuba. Urban Studies, 45(5-6), 1079-1096.

Baloglu, S. (1997). The relationship between destination images and sociodemographic and trip characteristics of international travellers. Journal of Vacation Marketing, 3(3), 221-233.

Baloglu, S., \& McCleary, K. W. (1999). A model of destination image formation. Annals of tourism research, 26(4), 868-897.

Chandler, G. N., \& Hanks, S. H. (1993). Measuring the performance of emerging businesses: A validation study. Journal of Business venturing, 8(5), 391-408.

Claver-Cortés, E., Pereira-Moliner, J., \& Molina-Azorín, J. F. (2009). Strategic groups and performance in the Spanish hotel sector. The Service Industries Journal, 29(7), 943-961.

Cronbach, L. J. (1951). Coefficient alpha and the internal structure of tests. psychometrika, 16(3), 297-334.

Crompton, J. L., Fakeye, P. C., \& Lue, C. C. (1992). Positioning: The example of the Lower Rio Grande Valley in the winter long stay destination market. Journal of Travel Research, 31(2), 20-26. 
Crompton, J. L. (2011). A theoretical framework for formulating non-controversial prices for public park and recreation services. Journal of Leisure Research, 43(1), 1-29.

Echtner, C. M., \& Ritchie, J. B. (1993). The measurement of destination image: An empirical assessment. Journal of travel research, 31(4), 3-13.

Ekinci, Y. (2003). An investigation of the determinants of customer satisfaction. Tourism Analysis, 8(2), 193-196.

Flynn, B. B., Schroeder, R. G., \& Sakakibara, S. (1994). A framework for quality management research and an associated measurement instrument. Journal of Operations management, 11(4), 339-366.

Garrigós, F. J., \& Palacios, D. (2005). Competitive strategies and firm performance: A study in the Spanish hospitality sector. Management Research, 2(3), 251-269.

Grant, R. (2004). Dirección Estratégica, conceptos, técnicas y aplicaciones (Vol. 4). Valladolid: Thompson.

Hair Jr, J. F., Anderson, R. E., Tatham, R. L., \& Black, W. C. (1995). Multivariate Data Analysis with Readings, Englewood Cliffs, NJ.: PrenticeHall.

Jönsson, C., \& Devonish, D. (2009). An exploratory study of competitive strategies among hotels in a small developing Caribbean state. International Journal of Contemporary Hospitality Management, 21(4), 491-500.

Kim, J., Ritchie, J R., \& McCormick, B. (2012). Development of a Scale to Measure Memorable Tourism Experiences. Journal of Travel Research, 51(1): 12.

McLennan, C. L., Ruhanen, L., Ritchie, B., \& Pham, T. (2010). Dynamics of destination development: Investigating the application of transformation theory. Journal of Hospitality \& Tourism Research, 1096348010390816.

Menguzzato, M., \& Renau J.J. (1991). La dirección estratégica de la empresa. Un enfoque innovador del Management. Editorial Ariel. España.

Mintzberg, H., \& ; Quinn, J. B. (1997). The strategy process: Concepts, contexts, Cases. 4sth edition. Prentice Hall.

Mintzberg, H., \& Lampel, J. (1999). Reflecting on the strategy process. Sloan Management Review, 40(3), 21-30.

Navas, J., \& Guerras, L. (2002). La dirección estratégica de la empresa: Teoría y aplicaciones. Tercera Edición. Editorial Civitas, SA, España.

Pappas, J. M., Flaherty, K. E., \& Hunt, C. S. (2007). The joint influence of control strategies and market turbulence on strategic performance in sales-driven organizations. Journal of Behavioral and Applied Management, 8(2), 141-164.

Oppermann, M. (1996). Convention destination images: analysis of association meeting planners' perceptions. Tourism management, 17(3), 175-182. 
Ortigueira, L. C., \& Gómez-Selemeneva, D. (2012). Innovation in Tourist Management through Critical Success Factors: A Fuzzy Map. In Soft Computing in Management and Business Economics (pp. 361-373). Springer Berlin Heidelberg.

Tavitiyaman, P., Quu Zhang, H., \& Qu, H. (2012). The effect of competitive strategies and organizational structure on hotel performance. International Journal of Contemporary Hospitality Management, 24(1), 140-159.

Porter, M. E. (1991). Towards a dynamic theory of strategy. Strategic management journal, 12(S2), 95-117.

Porter, M. E. (1996). What is strategy? Harvard Business Review, November/December, 61-78.

Powell, T. C. (1992). Strategic planning as competitive advantage. Strategic Management Journal, 13 (7), 551-558.

Reichel, A., \& Haber, S. (2005). A three-sector comparison of the business performance of small tourism enterprises: An exploratory study. Tourism Management, 26(5), 681-690.

Ritchie, J. R., Wing Sun Tung, V., \& Ritchie, R. J. (2011). Tourism experience management research: Emergence, evolution and future directions. International Journal of Contemporary Hospitality Management, 23(4), 419-438.

Romero, I. (2000). La calidad como factor de competitividad su trascendencia para el sector turístico cubano. Universidad de la Habana, Cuba.

Rumelt, R., Schendel, D., \& Teece, D. (1994). Fundamental issues in strategy: A research agenda. Harvard Business School Press. Boston.

Sainaghi, R. (2010). Hotel performance: state of the art. International Journal of Contemporary Hospitality Management, 22 (7), 920-52.

Siu-Loon H. (2007). Strategic Management: Formulation, Implementation, and Control in a Dynamic Environment. Journal of Management and Organization, 11: 141-164.

Spengler E. (2004). La rehabilitación del centro histórico de La Habana: Una obra esencialmente humana. Centro de Cultura Contemporánea de Barcelona, España.

Schroeder, T. (1996). The Relationship of Residents' Image of their State as a Tourist Destination and their Support for Tourism. Journal of Travel Research, 34 (4), 71-73.

Sheehan, L., Ritchie, J. B., \& Hudson, S. (2007). The destination promotion triad: Understanding asymmetric stakeholder interdependencies among the city, hotels, and DMO. Journal of Travel Research, 46(1), 64-74. 